\title{
A few concluding remarks
}

\author{
A. Baglin ${ }^{1}$ \\ ${ }^{1}$ LESIA, Observatoire de Paris, 92195 MEUDON, France
}

\begin{abstract}
Since the discovery of time variability in observed stellar quantities, as apparent magnitude and spectral characteristics, this phenomenon has been considered as a very important source of information to understand stars. Indeed, the global surface parameters as effective temperature, luminosity, and surface chemical composition tell a lot and have been extensively used in building internal structure theory. But this information is generally not sufficient as it is "degenerate", different internal physical conditions producing identical surface parameters. Dynamical phenomena, as intrinsic oscillations are the ideal indicators of these internal properties. They are present in many types of stars, with very different characteristics. The method and tools to observe them, as well as their potential of diagnostics in terms of internal structure are then very diverse.

In this symposium, the different aspects of variability studies and seismology have been presented, from the discovery of new objects and new effects, to the inference of the physical parameters of the interiors, with particularly difficult steps such as mode identification.
\end{abstract}

\section{The large population of variable stars}

Many classes of variables have been revisited, leading to new members of existing classes, to the discovery of new effects, based on progress of instrumentation, of time coverage, of the use of extensive data mining.

Variable stars as members of groups provide more complete information and can help interpretation. Several clusters have been revisited or studied for the first time. Some open clusters are very promising by their large content of Delta Scuti stars.

It is more and more easy to compare variables in galaxies of the local group to our own, allowing to study the effect of metallicity for instance, which is particularly important for classes of variables where it is predicted that metallicity is the driving factor of the oscillations, as e.g. in $\beta$ Cephei stars and SPBs.

Statistical studies provide also some hints for interpretation. They benefit from large surveys, essentially MACHO on the Magellanic Clouds and OGLE for the galactic bulge.

Observations over long periods allow to detect variations of pulsational properties on different time scales, which are not yet understood in general.

Using large telescopes with high resolution spectrographs, detailed spectroscopic studies are revisiting well known behaviors like for instance the Blazhko effect in RR Lyrae stars.

The long standing question about the coexistence of both variable and non variable stars in the same region of the HR diagram is still pending in most cases. For instance, abundance differences observed in $\lambda$ Bootis stars do not provide all the necessary clues. 


\section{Mode identification}

One of the key issues to extract the information from any seismic data is the ability to identify the mode of pulsation corresponding to an observed frequency. Unfortunately, the structure of the power spectrum is generally much more complicated than the solar comb. Spectra are often coarse and non equidistant. Except in white dwarfs where rotational splitting allows to determine the I value, there is no indisputable identification, even in the very famous $\delta$ Scuti star FG Vir, where more than 20 modes have been detected.

Since many years it has been proposed to use the phase differences and amplitude ratios of the oscillation in different colours to test the horizontal structure of the modes ( 1 and $\mathrm{m}$ values). It is now recognized that this method requires a comparison to refined modelling of the outer layers, including non-adiabatic effects and convection/pulsation interaction in cool stars. In $\delta$ Scuti and $\gamma$ Doradus stars, the perturbation of the eigenfunctions due to rotation has also to be taken into account. This technique is very promising, but a lot more work has to be done to be able to perform an unambiguous determination of the I value; the quality of the data has also to be improved.

In eclipsing binaries, the transit phase can be used to map the stellar surface and detect the geometrical properties of the oscillation.

In rapidly rotating stars with wide line profiles, surface imaging techniques are promising tools to map the oscillation on the disk.

\section{Seismic interpretation}

Stellar seismology is in general more difficult to handle than helioseismology. The old slowly rotating Sun, with its radiative core has the simplest oscillation behavior one can think of!

The generalization of techniques used in the solar case to slightly different types of models gives valuable tools to understand the variation of the stellar structure with mass and age in the solar vicinity.

But, it is already possible to generalize the seismology techniques to quite different classes of stars. The discovery of a few pulsating pre-main sequence Delta Scuti stars stimulated both observational and theoretical studies, aiming at determining the behavior of stars in their contraction phase. Also, in large amplitude pulsators (HADS), with a few modes, the classical method consisting in using period ratios to determine some global properties can nicely be used.

In white dwarfs, long term variations of frequencies interpreted as a signature of the cooling process provide some insight on the state of matter in the central regions and test the existence of axions.

Among the wide efforts aiming at using the seismic signatures (frequency, amplitude, and eventually life-time of the detected modes) to infer stellar properties, hydrodynamical simulations will bring some new insight in mode selection effects, in excitation processes, in surface effects...

Another important difficulty in seismic interpretation is due to the uncertainties on the fundamental parameters of distant stars. This problem evidently and thankfully did not exist for the Sun. Efforts have to be made on that subject, dealing with detailed physics of the stellar atmospheres, to be able for instance to compare measured and computed "effective temperatures" at the level of a few tens of degrees. In this respect, the advent of interferometry with large telescopes may lead, at least for bright stars, to radius determinations of sufficient accuracy to be useful in seismic studies. Working with homogeneous groups as clusters will help, but will not cover all types of stars. 


\section{Ground based observations and networks}

Europe has a wide variety of instruments and the number of astronomers involved in these activities is quite large.

In photometry, long duration coordinated campaigns have proven to be very efficient in deciphering the spectra of White dwarfs, for instance. And it is evidently this type of observations which is required to progress in the improvement of our knowledge of the pulsation behavior of many objects.

As one of the major requirements is a high duty cycle over many days, it implies coordinated networks with a reasonable coverage in longitude in very good photometric sites. Each such a campaign is a huge effort. Robotic telescopes are developing rapidly. Coordinated in networks and equipped with instrumentation as homogeneous as possible, they can help in reducing the work load. However it remains necessary to select carefully the targets, and to coordinate activities of many countries in order not to waste efforts and telescope time.

The spectroscopic technique has already been very successful in detecting solar-like oscillations in a few bright stars. Surface imaging in fast rotators is able to detect oscillations with large $m$ values. It requires access to large telescopes, equipped with stable and precise spectrographs. Once again the ideal situation would be to operate a world-wide homogeneous network, but presently it is out of the possibilities.

\section{Space observations}

Space, in principle, overcomes many of the difficulties of the ground observations: transparency, stability and continuity though it is clear that it will never supersede them.

Space projects dedicated to stellar variability have been proposed since more than 20 years and only recently selection committees have considered them positively.

Due to the required size of the instrument, and the need to access all types of stars, including fast rotators, the photometric technique has been preferred up to now.

MOST: the pioneer
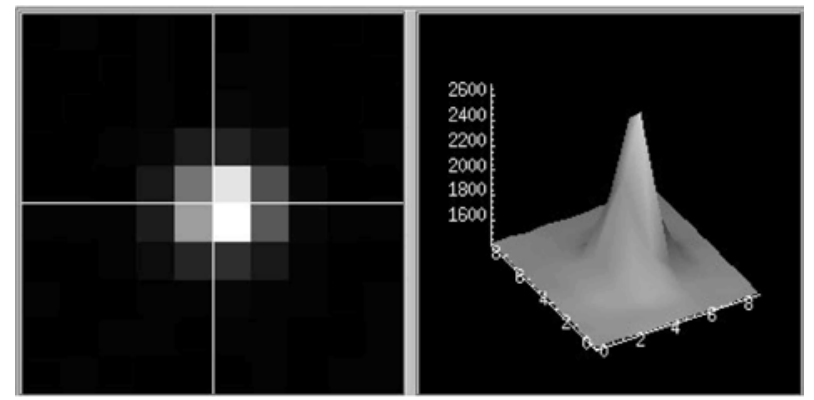

Figure 1: First image of the MOST star tracker

The Canadian suitcase-sized microsatellite MOST has been launched successfully on June 30th by Rockot into a heliosynchronous low earth orbit at $820 \mathrm{~km}$.

Its $15 \mathrm{~cm}$ collector will observe a few bright stars $\left(m_{v} \leq 6\right)$, of different characteristics for continuous periods of 30 up to 58 days.

News can be found at http://www.astro.ubc.ca/MOST/index.html 


\section{COROT and EDDINGTON}

It is in Europe, and particularly in France, that the first space projects have been proposed in the 80s.

Unfortunately after the crash of the MARS96 spacecraft, with the small experiment EVRIS on board, new opportunities were scarce. In the framework of the national Danish program the Roemer/Mons project has been studied till the end of Phase B, but then stopped. The CNES programme of "small missions" preselected COROT in 1994, which since then has succeeded to overcome all the selections and budget difficulties.
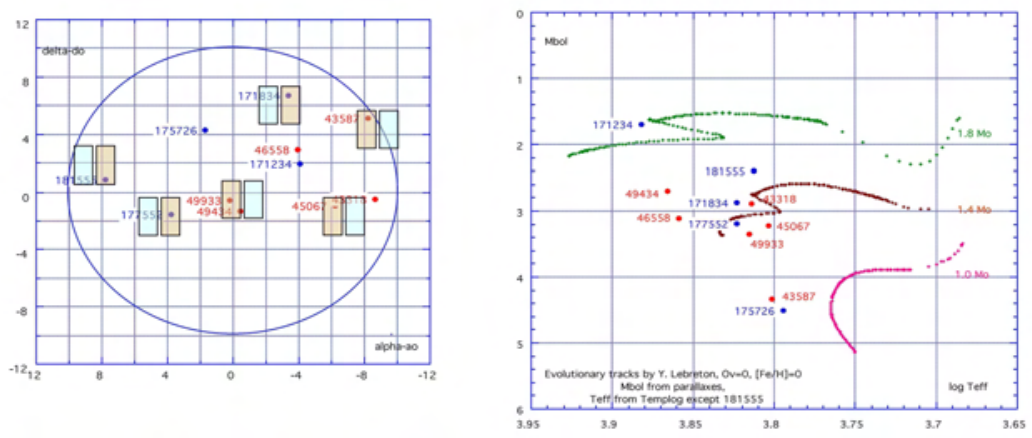

Figure 2: A preliminary set of candidates for the principal targets of the seismology programme of COROT, as seen on the sky, with the focal plane, and in the HR diagram. These targets are the brightest ones, and will be followed continuously for 150 days. Ten times more objects around these ones will be observed at the same time.

COROT is now in phase C, to be launched in early 2006.

It has a specific seismology programme of very high accuracy, observing 50 stars for 150 days and 100 for 20 days. A very large number of faint stars $(180000)$ will be observed with a lower accuracy and lower time sampling, but providing continuous time series of extreme importance for variability studies.

More information can be found at http://corot.astrsp-mrs.fr

With COROT, France and Europe take the floor. But this European leadership has to be maintained, through a second generation project, using the expertise acquired for COROT.

The project EDDINGTON is this second step in ultra-high precision photometry from space. As Corot it will have two major programmes: one dedicated to a general survey of the seismic properties of stars, the other one to the discovery of planetary systems.

When writing this paper, the decision to implement Eddington has not been taken. The financial difficulties of the science Programme of ESA have lead the Science Programme Committee to propose an affordable version of the "Cosmic Vision" programme which does not include Eddington, though considering its extreme scientific importance. Let's hope that a solution will be found to be able to overcome this disastrous situation and allow the development of Eddington in due time to remain a major actor in the international game of ultra-high precision stellar photometry, after COROT. 


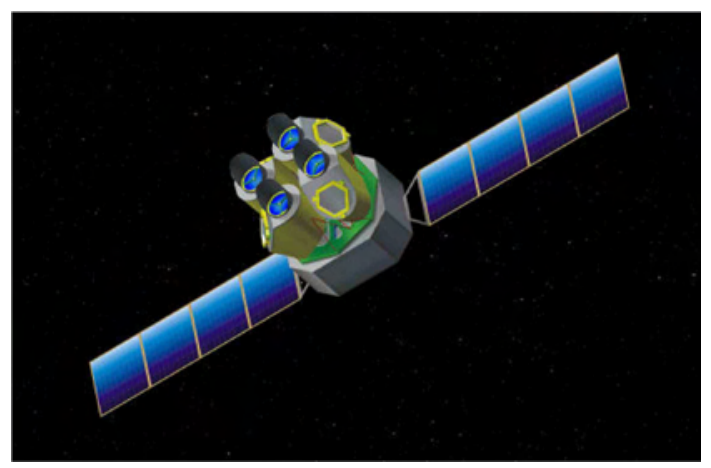

Figure 3: An artistic view of Eddington

\section{The European Network ENEAS}

As demonstrated in this symposium and also browsing literature, Europe plays a major role in stellar variability and seismology studies.

As the different steps of a seismic study need strong efforts, very broad and different expertise, the European community has created a network of scientists and laboratories called ENEAS in October 2002.

As described by Aerts et al. (2003), ENEAS is a tool to strengthen Europe's position in this field, to prepare the European community to exploit the new ground based instrumentation and the space missions by exchange of expertise and training, and to coordinate efforts. Presently 256 scientists from 43 Institutions have joined the group.

A web site http://www.eneas.info has been set up to provide a natural and vivid link between the participants, even before any formal support is obtained.

\section{References}

Aerts, C., Baglin, A. et al. 2003, Second Eddington Workshop ESA-SP, in press.

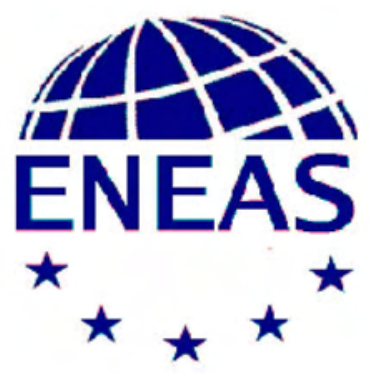

Figure 4: The ENEAS logo 